\title{
REPRESENTATION THEOREMS FOR CERTAIN BOOLEAN ALGEBRAS
}

\author{
R. S. PIERCE
}

1. Introduction. Recently, three papers $[6 ; 2$ and 4$]$ on the problem of representing an $\alpha$-complete Boolean algebra as an $\alpha$-homomorph of an $\alpha$-field have been published. The present paper can be considered as a fourth contribution to this problem of characterizing $\alpha$-representable algebras. We generalize the criterion of Scott and Tarski [4] for $\alpha$-representability and study the Boolean algebras satisfying these conditions. This program seems to be justified. Our results not only generalize the work in the papers listed above, but also lead to representations of a different kind than have been considered heretofore (e.g., 3.9 and 3.12 below). Methodologically, this paper is closely related to the work of R. Sikorski (in [5] for example). We use topological tools furnished by the Stone representation theorem, whereas Smith [6] and Chang [2] proceed along algebraic lines, and Scott and Tarski [4] adopt a metamathematical viewpoint.

2. Preliminaries. Throughout the paper, $\alpha$ and $\beta$ denote infinite cardinal numbers. We write $\alpha \bigvee \beta$ for $\max [\alpha, \beta]$. The symbol $\infty$ is used as if it were a cardinal exceeding all other cardinal numbers. For any $\alpha$, define $\alpha+$ to be the least cardinal exceeding $\alpha$. The terms. $\alpha$-ideal and $\alpha$-field are used in the usual way, that is, the operations in these structures enjoy closure up to and including the power $\alpha$. In particular, the term complete field means the field of all subsets of some set. The notions of $\alpha$-homomorphism, $\alpha$-isomorphism and $\alpha$ subalgebra will have special meanings which are explained in 3.1 below.

Lattice operations of join, meet, inclusion and complement are designated by $\vee, \wedge$, $\leqq$ and (') respectively. Corresponding set operations are represented by rounded symbols: $\cup, \cap, \subseteq$ and $\left(^{(}\right)$. The symbols 0 and $\overline{0}$ will always stand for the zero of a Boolean algebra, while $u$ and $\bar{u}$ denote the unit element.

The empty set is designated by $\varnothing$. If $S$ and $T$ are sets, $T-S$ is the set of elements in $T$, but not in $S$. If $h$ is a mapping of $S$ into $T$, and if $X \subseteq S, Y \subseteq T$, we denote by $h(X)$ the set $\{h(x) \mid x \in X\}$ and by $h^{-1}(Y)$ the set $\{x \in X \mid h(x) \in Y\}$. The symbol $|S|$ designates the cardinality of the set $S$.

Presented to the Society January 30, 1958; received by the editors September 28, 1957. 
We will of ten use the abbreviation B.A. for Boolean algebra. If $A$ is a subset of the Boolean algebra $B$, then the least upper bound of $A$ in $B$, if it exists, is denoted either l.u.b. $A$, or $\mathrm{V}_{\sigma \in S} a_{\sigma}$, where $A$ $=\left\{a_{\sigma} \mid \sigma \in S\right\}$. A subset $A$ of a B.A. is called an $\alpha$-cover if $|A| \leqq \alpha$ and 1.u.b. $A=u$. If also $A$ is disjoined, that is, $a \wedge b=0$ for distinct $a$ and $b$ in $A$, then $A$ is called an $\alpha$-partition. If $A_{1}, \cdots, A_{n}$ are $\alpha$ partitions, define $A_{1} \wedge \cdots \wedge A_{n}=\left\{a_{1} \wedge \cdots \wedge a_{n} \mid a_{i} \in A_{i}\right\}$. By the infinite distributive law for Boolean algebras (see $[1$, p. 165]), $A_{1} \wedge \cdots \wedge A_{n}$ is also an $\alpha$-partition. A subset $\bar{A}$ of a B.A. is said to refine the subset $A$ if, for any $\bar{a} \in \bar{A}$, there exists $a \in A$ with $\bar{a} \leqq a$. It is clear in this case that if $\bar{A}$ is a cover, so is $A$.

The Boolean space $X$ of a Boolean algebra $B$ is the set of all prime ideals $P$ of $B$, topologized by taking the sets

$$
X(a)=\{P \in X \mid a \notin P\}, \quad a \in B
$$

as a basis for the open sets. It is well known [8] that $X$ is a totally disconnected, compact Hausdorff space with this topology. Also, the mapping $a \rightarrow X(a)$ is a lattice isomorphism of $B$ onto the B.A. of all open-and-closed subsets of $X$.

Definition 2.1. Let $B$ be a B.A. and let $X$ be the Boolean space of $B$. Then $T \subseteq X$ is called $\alpha$-nowhere dense ( $\alpha$-disbursed) if there is an $\alpha$-cover (resp. $\alpha$-partition) $A$ of $B$ such that $T \subseteq I(A)=\left(\bigcup_{a \in A} X(a)\right)$.

Definition 2.2. A Boolean algebra $B$ is a $C_{\alpha \beta^{-}}$algebra (resp. $D_{\alpha \beta^{-}}$ algebra) if no $\alpha$-union of $\beta$-nowhere dense sets (resp. $\beta$-disbursed sets) of the Boolean space of $B$ contains a nonempty open subset of the space.

Every B.A. satisfies $C_{\alpha_{\infty}}$ if $\alpha=\boldsymbol{\aleph}_{0}$, since no nonempty compact open set is a countable union of nowhere dense subsets. Also, it is easy to see that every $(\alpha, \beta)$-distributive B.A. (see [7]) satisfies $C_{\alpha \beta}$. In particular, any complete field has the property $C_{\infty \infty \infty}$.

Taking account of the above remarks on Boolean spaces, the properties $C_{\alpha \beta}$ and $D_{\alpha \beta}$ can be formulated algebraically as follows: if $\left\{A_{\sigma} \mid \sigma \in S\right\},|S| \leqq \alpha$, is a family of $\beta$-covers $\left(\beta\right.$-partitions for $\left.D_{\alpha \beta}\right)$ and $b \neq u$, then there is a prime ideal $P$ of $B$ with $b \in P$ and $P \nsupseteq A_{\sigma}$ for all $\sigma \in S$. In case $\alpha=\beta$, this is precisely the criterion of Scott and Tarski for $\alpha$-representability.

Lemma 2.3. Let $B$ be a B.A., $X$ its Boolean space and $T \subseteq X$.

(a) If $T$ is $\alpha$-disbursed, then $T$ is $\alpha$-nowhere dense;

(b) if $B$ has the property $R_{\alpha}$ : every $\alpha$-covering can be refined by an $\alpha$-partition, then any $\alpha$-nowhere dense set is $\alpha$-disbursed;

(c) every B.A. has the property $R_{\infty}$; 
(d) if $B$ is $\alpha$-complete, then $B$ has the property $R_{\alpha+}$;

(e) if $B$ satisfies $C_{\alpha \beta}$ (resp. $\left.D_{\alpha \beta}\right)$ and $\gamma \leqq \alpha, \delta \leqq \beta$, then $B$ satisfies $C_{\gamma \delta}\left(D_{\gamma \delta}\right)$;

(f) every $C_{\alpha \beta}$-algebra is a $D_{\alpha \beta}$-algebra; every $D_{\alpha \beta}$-algebra with property $R_{\beta}$ is a $C_{\alpha \beta}$-algebra; in particular, $D_{\alpha \infty}$ is equivalent to $C_{\alpha \infty}$ and any $\beta$-complete $D_{\alpha \beta}$-algebra is a $C_{\alpha \beta+}$-algebra.

Proof. Only (c) and (d) require any comment. If $A$ is an $\infty$-cover of $B$, let $\bar{A}$ be a maximal disjointed subset of $B$ refining $A$. It is easy to see that maximality implies l.u.b. $\bar{A}=u$, so (c) follows. To prove (d), let the $\alpha+$-cover $A$ be indexed by the ordinals of cardinality $<\alpha+: A=\left\{a_{\xi}|| \xi \mid<\alpha+\right\}$. Then $\bar{A}=\left\{b_{\xi}|| \xi \mid<\alpha+\right\}$, where $b_{\xi}$ $=a_{\xi} \wedge\left(\bigvee_{\eta<\xi} a_{\eta}\right)^{\prime}$, is an $\alpha+$-partition which refines $A$.

\section{3 . The representation theorem.}

Definition 3.1. Let $B$ and $\bar{B}$ be Boolean algebras and $h: B \rightarrow \bar{B}$ a homomorphism. We call $h$ an $\alpha$-homomorphism (resp. weak $\alpha$ homomorphism) if, whenever $A \subseteq B,|A| \leqq \alpha$ and $b=1$.u.b. $A$ exists (and, for the weak $\alpha$-homomorphism, $A$ is disjointed), then

$$
h(b)=\text { l.u.b. } h(A) \text {. }
$$

An $\alpha$-homomorphism which is one-to-one (but not necessarily onto) is termed an $\alpha$-isomorphism. A subalgebra $B$ of $\bar{B}$ is called an $\alpha$ subalgebra if the injection mapping of $B$ into $\bar{B}$ is an $\alpha$-isomorphism.

It is clear from this definition that $B$ is isomorphic to an $\alpha$-subalgebra of $\bar{B}$ if and only if there is an $\alpha$-isomorphism of $B$ into $\bar{B}$. If $B$ and $\bar{B}$ are $\alpha$-complete, the concepts of $\alpha$-homomorphism and $\alpha$ subalgebra in 3.1 are the usual ones. Moreover, the $\alpha$-homomorphic image of an $\alpha$-complete B.A. is $\alpha$-complete. However, in the absence of $\alpha$-completeness, the notions in 3.1 are somewhat novel. For example, the B.A. of all finite subsets and their complements in a set $S$ is an $\infty$-subalgebra of the B.A. of all subsets of $S$.

Lemma 3.2. Let $h: B \rightarrow \bar{B}$ be a homomorphism. Then $h$ is an $\alpha$ homomorphism (resp. weak $\alpha$-homomorphism) if and only if for any $\alpha$-cover ( $\alpha$-partition) $A$, the image $h(A)$ is an $\alpha$-cover of $\bar{B}$.

Proof. This condition is clearly necessary. Hence, suppose $A \subseteq B$, $|A| \leqq \alpha, b=1$.u.b. $A$ (and $A$ is disjointed, for the weak case). Let $A_{1}=A \cup\left\{b^{\prime}\right\}$. Then $A_{1}$ is an $\alpha$-cover (resp. $\alpha$-partition), so by hypothesis, $h\left(A_{1}\right)$ is an $\alpha$-cover. Evidently $h(b) \geqq h(a)$ for all $a \in A$, so $h(b)$ is an upper bound of $h(A)$. If $\bar{c} \in \bar{B}$ satisfies $\bar{c} \geqq h(a)$ for all $a \in A$, then $h\left(b^{\prime}\right) \bigvee \bar{c} \geqq$ l.u.b. $h\left(A_{1}\right)=\bar{u}$. Hence $\bar{c} \geqq\left(h\left(b^{\prime}\right)\right)^{\prime}=h(b)$. This proves that $h(b)=$ l.u.b. $h(A)$. 
CoRollary 3.3. If $B$ has the property $R_{\alpha}$, then every weak $\alpha$-homomorphism of $B$ is an $\alpha$-homomorphism.

Proof. Suppose $A$ is an $\alpha$-cover. Let $\bar{A}$ be an $\alpha$-partition refining $A$. By assumption, $h(\bar{A})$ is an $\alpha$-cover. But $h(\bar{A})$ refines $h(A)$, so $h(A)$ is an $\alpha$-cover. Thus, $h$ is an $\alpha$-homomorphism.

Lemma 3.4. Let $A$ be an $\alpha$-partition of $B$. Suppose $F \subseteq X$ has the property that $F \cap X(a)$ is $\alpha$-disbursed for all $a \in A$. Then $F$ is $\alpha$-disbursed.

Proof. For each $a \in A$, there is an $\alpha$-partition $A_{a}$ such that $F \cap X(a) \subseteq I\left(A_{a}\right)$. Define

$$
\bar{A}=\bigcup_{a \in A}\left\{a \wedge b \mid b \in A_{a}\right\} .
$$

Clearly, $\bar{A}$ is an $\alpha^{2}=\alpha$-partition of $B$. We will show that $F \subseteq I(\bar{A})$.

By definition,

$$
\begin{aligned}
I(\bar{A})^{c} & =\bigcup_{a \in A}\left(U\left\{X(a \wedge b) \mid b \in A_{a}\right\}\right) \\
& =\bigcup_{a \in A}\left(X(a) \cap\left(\bigcup\left\{X(b) \mid b \in A_{a}\right\}\right)\right)=\bigcup_{a \in A} X(a) \cap I\left(A_{a}\right)^{c} .
\end{aligned}
$$

Thus, $F \cap I(\bar{A})^{c}=\bigcup_{a \in A} F \cap X(a) \cap I\left(A_{a}\right)^{c}=\varnothing$, since $F \cap X(a) \subseteq I\left(A_{a}\right)$ for all $a \in A$. Therefore $F \subseteq I(\bar{A})$.

Definition 3.5. Let $B$ be a Boolean algebra and $I$ an ideal of $B$. A homomorphism $h$ of a Boolean algebra $\bar{B}$ into $B / I$ will be called factorable if there is a homomorphism $g$ of $\bar{B}$ into $B$ such that $h=p \circ g$, where $p: B \rightarrow B / I$ is the natural projection.

Theorem 3.6. Let $B$ be a $D_{\alpha \beta}$-algebra. Then there is a factorable, weak $\beta$-isomorphism of $B$ into a Boolean algebra $\bar{B}$ which is an $\alpha$ homomorph of a complete field of sets.

Proof. Let $X$ be the Boolean space of $B$. Denote by $\mathfrak{B}$ the Boolean algebra of all subsets of $X$. Let $\Im$ be the $\alpha$-ideal of $\mathfrak{B}$ generated by the $\beta$-disbursed sets of $X$. Then $\Im$ consists of all subsets of $X$ which are contained in some $\alpha$-union of $\beta$-disbursed sets. Let $\bar{B}=\mathfrak{B} / \Im$, so that $\bar{B}$ is an $\alpha$-homomorph of a complete field. For $a \in B$ define $h(a)$ $=p(X(a))$, where $p$ is the natural homomorphism of $\mathfrak{B}$ onto $\mathfrak{B} / \mathfrak{Y}$. Clearly $h$ is a factorable homomorphism. If $h(a)=\overline{0}$ for some $a \neq 0$, then $X(a) \in \mathfrak{B}$, i.e., $X(a)$ is contained in an $\alpha$-union of $\beta$-disbursed sets. But $X(a)$ is a nonempty open set and the property $D_{\alpha \beta}$ says that no $\alpha$-union of $\beta$-disbursed sets contains a nonempty open set. Hence, $h$ is one-to-one. It remains to show that $h$ is a weak $\beta$-iso- 
morphism. By 3.2, we have to prove that if $A$ is a $\beta$-partition of $B$, then $h(A)$ is a $\beta$-cover of $\bar{B}$.

Let $F \in \Im$ and assume that $p(F) \neq \overline{0}$. To show that $h(A)$ is a $\beta$ cover, we will prove that the arbitrary nonzero element $p(F)$ of $\bar{B}$ meets some element of $h(A)$. Suppose otherwise: $p(F) \wedge h(a)=\overline{0}$ for all $a \in A$. That is, $F \cap X(a) \in \Im$ for all $a \in A$. Then $F \cap X(a)$ $\subseteq \mathrm{U}_{\tau \in T} I\left(A_{a \tau}\right)$, where $T$ is an index set of cardinality $\alpha$ and $A_{a \tau}$ is a $\beta$-partition. Define $F_{\tau}=\bigcup_{a \in A}\left(F \cap X(a) \cap I\left(A_{a \tau}\right)\right)$. Since $A$ is a partition, $F_{\tau} \cap X(a)=F \cap X(a) \cap I\left(A_{a \tau}\right) \subseteq I\left(A_{a \tau}\right)$, that is, $F_{\tau} \cap X(a)$ is $\beta$ disbursed for all $a \in A$. By 3.4, $F_{\tau}$ is $\beta$-disbursed. Hence

$$
\begin{aligned}
\bigcup_{\tau \in T} F_{\tau} & =\bigcup_{\tau \in T} \bigcup_{a \in A}\left(F \cap X(a) \cap I\left(A_{a \tau}\right)\right)=\bigcup_{a \in A}\left(\bigcup_{\tau \in T}\left(F \cap X(a) \cap I\left(A_{a \tau}\right)\right)\right) \\
& =\bigcup_{a \in A}\left((F \cap X(a)) \cap\left(\bigcup_{\tau \in T} I\left(A_{a \tau}\right)\right)\right)=\bigcup_{a \in \boldsymbol{A}} F \cap X(a) \\
& =F \cap\left(\bigcup_{a \in A} X(a)\right)=F \cap I(A)^{c}
\end{aligned}
$$

is an $\alpha$-union of $\beta$-disbursed sets. Thus $F \cap I(A)^{c} \in \Im$. But also $F \cap I(A) \in \Im$, since $I(A)$ is $\beta$-disbursed. Therefore $F \in \Im$. Since this contradicts the assumption $p(F) \neq \overline{0}$, the proof is complete.

Corollary 3.7. If $B$ is a $\beta$-complete $C_{\alpha \beta}$-algebra, then $B$ is isomorphic to a $\beta$-subalgebra of an $\alpha$-homomorph of a complete field.

Proof. By 3.6 and 2.3 (f), there is a weak $\beta$-isomorphism of $B$ into $\bar{B}$, an $\alpha$-homomorph of a complete field. But 2.3 and 3.3 imply that this weak $\beta$-isomorphism is actually a $\beta$-isomorphism.

Corollary 3.8. An $\alpha$-complete $C_{\infty \alpha}$-algebra is isomorphic to an $\alpha$-field of sets.

Proof. Apply 3.7 and notice that any $\infty$-homomorph of a complete field is a complete field.

Since every B.A. satisfies $C_{\alpha \infty}$ (and hence $C_{\alpha \alpha}$ ) is $\alpha=\boldsymbol{\aleph}_{0}$, the following is a special case of 3.7.

CoRollary 3.9. Every $\boldsymbol{\aleph}_{0}$-complete Boolean algebra is isomorphic to an $\boldsymbol{\aleph}_{0}$-subalgebra of an $\boldsymbol{\aleph}_{0}$-homomorph of a complete field of sets.

REMARK. It is illuminating to compare 3.9 with the theorem of Loomis [3]: every $\boldsymbol{\aleph}_{0}$-complete B.A. is isomorphic to an $\boldsymbol{\aleph}_{0}$-homomorph of an $\boldsymbol{\aleph}_{0}$-subalgebra of a complete field. It is easy to deduce Loomis's theorem from 3.9 and conversely. Indeed, Loomis's theorem is a special case of our next result. 
Corollary 3.10. Suppose $B$ is an $\alpha$-complete $C_{\alpha \alpha}$-algebra. Then $B$ admits a factorable isomorphic representation as an $\alpha$-homomorph of. an $\alpha$-field.

Proof. By 3.6, 2.3(d) and 3.3, there exists a factorable $\alpha$-isomorphism $h: B \rightarrow \mathfrak{B} / \Im$, where $\mathfrak{B}$ is a complete field and $\Im$ is an $\alpha$-ideal, say $h=p \circ g, g: B \rightarrow \mathfrak{B}$ and $p: \mathfrak{B} \rightarrow \mathfrak{B} / \Im$ (the natural homomorphism). Let $\mathfrak{F}=p^{-1}(h(B))$. Since $h$ and $p$ are $\alpha$-homomorphisms and $B$ is $\alpha$-complete, $\mathfrak{F}$ is an $\alpha$-field. Clearly $\mathfrak{F}$ contains $g(B)$. Thus, $h: B \rightarrow p(\mathfrak{F})$ is a factorable isomorphism of $B$ into an $\alpha$-homomorph of an $\alpha$-field.

Corollary 3.11. If $B$ is a $C_{\alpha \infty}$-algebra, then there is an $\infty$-isomorphism of $B$ into an $\alpha$-homomorph of a complete field.

Proof. By 2.3(c) and (f), 3.3 and 3.6.

In particular, the remark following 3.8 implies the following.

CoROllaRy 3.12. Every B.A. is isomorphic to an $\infty$-subalgebra of an $\aleph_{0}$-homomorph of a complete field of sets.

This last corollary clearly contains 3.9 as a special case. By examing the proof of 3.6 , we see that the representation in 3.12 can be described more precisely as an $\infty$-isomorphism of a Boolean algebra $B$ into the quotient of the B.A. of all subsets of the Boolean space of $B$, modulo the sets of the first category in this space.

\section{The converse theorem.}

Lemma 4.1. Let $B$ be a $\beta$-complete B.A. and let $I$ be an ideal of $B$. Denote by $p$ the natural homomorphism $B \rightarrow B / I$. Suppose $A$ is a disjointed subset of $B$ with $|A| \leqq \beta$, for which $p(A)$ is a cover of $B / I$. Then there exists $w \in I$ such that $(A-A \cap I) \cup\{w\}$ is a $\beta$-partition of $B$.

Proof. Let $w=(\text { l.u.b. }(A-A \cap I))^{\prime}$. Since $A$ is disjointed, $(A-A \cap I) \cup\{w\}$ is a $\beta$-partition of $B$. We only have to show that $w \in I$. To do so, just note $p(w)=p(w) \wedge p(u)=p(w) \wedge$ l.u.b. $p(A)$ $=p(w) \wedge$ l.u.b. $p(A-A \cap I)=$ l.u.b. $\{p(w \wedge a) \mid a \in A-A \cap I\}$ $\leqq p($ l.u.b. $\{w \wedge a \mid a \in A-A \cap I\})=p(w \wedge$ l.u.b. $(A-A \cap I))=p(0)$.

Corollary 4.2. Under the same conditions as in 4.1, there exists a $\beta$-partition $A_{1}$ of $B$ such that $p\left(A_{1}\right)=p(A)$.

Proof. If $A \cap I \neq \varnothing$. Let $A_{1}=(A-A \cap I) \cup\{w\}$. Otherwise, choose any $a \in A$ and define $A_{1}=(A-\{a\}) \cup\{a \vee w\}$.

Theorem 4.3. Suppose $B$ is an $\alpha \bigvee \beta$-complete Boolean algebra satisfying $D_{\alpha \beta}$. Assume $I$ is an $\alpha$-ideal of $B$ and denote by $p$ the natural 
homomorphism of $B$ on $B / I$. Let $\bar{B}$ be a B.A. and $h: \bar{B} \rightarrow B / I$ a weak $\beta$-isomorphism with the property

$L_{\beta}:$ if $\bar{A}$ is a $\beta$-partition of $\bar{B}$, there exists a $\beta$-partition $A$ of $B$ such that $h(\bar{A})=p(A)$.

Then $\bar{B}$ satisfies $D_{\alpha \beta}$.

Proof. Let $\left\{\bar{A}_{\sigma} \mid \sigma \in A\right\}$ be a family of $\beta$-partitions of $\bar{B}$ with $|S| \leqq \alpha$. Suppose $\bar{b} \neq \overline{0}$ in $\bar{B}$. We must show that there is a prime ideal $\bar{P}$ of $\bar{B}$ with $b \notin \bar{P}$ and $\bar{P} \nsupseteq \bar{A}_{\sigma}$ for all $\sigma \in S$.

By $L_{\beta}$, it is possible to choose $\beta$-partitions $A_{\sigma}$ of $B$ such that $p\left(A_{\sigma}\right)=h\left(\bar{A}_{\sigma}\right)$. Also, because $p$ is onto, we can find $b \in B$ such that $p(b)=h(b)$. Since $b \neq \overline{0}$ and $h$ is an isomorphism, $b \notin I$.

For $\sigma_{1}, \cdots, \sigma_{n}$ in $S$, let $J\left(\sigma_{1}, \cdots, \sigma_{n}\right)$ be all elements in $I$ which are of the form $b \wedge a_{1} \wedge \cdots \wedge a_{n}$, with $a_{i} \in A_{\sigma_{1}}$. The cardinality of $J\left(\sigma_{1}, \cdots, \sigma_{n}\right)$ is evidently $\leqq \beta$, so we can define $w\left(\sigma_{1}, \cdots, \sigma_{n}\right)$ $=$ l.u.b. $J\left(\sigma_{1}, \cdots, \sigma_{n}\right)$. Applying 4.1 to the $\beta$-partition $A=\left\{b, b^{\prime}\right\}$ $\wedge A_{\sigma_{1}} \wedge \cdots \wedge A_{\sigma_{n}}$ shows that $w\left(\sigma_{1}, \cdots, \sigma_{n}\right) \in I$. (Note $p(A)$ $=\left\{h(b), h\left(\bar{b}^{\prime}\right)\right\} \wedge h\left(\bar{A}_{\sigma_{1}}\right) \wedge \cdots \wedge h\left(\bar{A}_{\sigma_{n}}\right)$ is a cover of $\left.B / I\right)$.

Put $z=$ l.u.b. $\left\{w\left(\sigma_{1}, \cdots, \sigma_{n}\right) \mid \sigma_{1}, \cdots, \sigma_{n} \in S, n=1,2, \cdots\right\}$. The cardinality of the set of these $w$ 's being $\leqq \alpha$, it follows that $z \in I$. In particular, $b \wedge z^{\prime} \neq 0$.

Since $B$ satisfies $D_{\alpha \beta}$, there exists a prime ideal $P$ of $B$ with $P \in X\left(b \wedge z^{\prime}\right)$ and $P \in \mathrm{U}\left\{X(a) \mid a \in A_{\sigma}\right\}$ for all $\sigma \in S$. Hence, for each $\sigma$, we can find $a_{\sigma} \in A_{\sigma}$ with $P \in X\left(a_{\sigma}\right)$. Because $p\left(A_{\sigma}\right)=h\left(\bar{A}_{\sigma}\right)$, there is a unique element $\bar{a}_{\sigma} \in \bar{A}_{\sigma}$ satisfying $h\left(\bar{a}_{\sigma}\right)=p\left(a_{\sigma}\right)$. Consider the sets $X\left(h\left(\bar{a}_{\sigma}\right)\right)=X\left(p\left(a_{\sigma}\right)\right)$. These have the finite intersection property with $X(h(b))=X(p(b))$. For otherwise it would be possible to find $\sigma_{1}, \cdots, \sigma_{n}$ in $S$ such that $X(p(b)) \cap X\left(p\left(a_{\sigma_{1}}\right)\right) \cap \cdots \cap X\left(p\left(a_{\sigma_{n}}\right)\right)$ $=\varnothing$. Thus $b \wedge a_{\sigma_{1}} \wedge \cdots \wedge a_{\sigma_{n}} \in I$, so by definition, $b \wedge a_{\sigma_{1}} \wedge \cdots \wedge a_{\sigma_{n}}$ $\leqq w\left(\sigma_{1}, \cdots, \sigma_{n}\right) \leqq z$. Equivalently, $b \wedge z^{\prime} \wedge a_{\sigma_{1}} \wedge \cdots \wedge a_{\sigma_{n}}=0$. But this is impossible since $P \in X\left(b \wedge z^{\prime}\right) \cap X\left(a_{\sigma_{1}}\right) \cap \cdots \cap X\left(a_{\sigma_{n}}\right)$ $=X\left(b \wedge z^{\prime} \wedge a_{\sigma_{1}} \wedge \cdots \wedge a_{\sigma_{n}}\right)$.

It follows that in the Boolean space of $\bar{B}$, the sets $X\left(\bar{a}_{\sigma}\right)$ have the finite intersection property with $X(b)$. By compactness, there is a prime ideal $\bar{P}$ of $\bar{B}$ with $\bar{P} \in X(\bar{b}) \cap \bigcap_{\sigma \in S} X\left(\bar{a}_{\sigma}\right)$. Thus $b \in \bar{P}$ and $\bar{P} \nsupseteq \bar{A}_{\sigma}$ for all $\sigma \in S$. This is the desired result.

If the mapping $h: \bar{B} \rightarrow B / I$ in 4.3 is not only a $\beta$-isomorphism, but is also factorable, then $L_{\beta}$ is automatically satisfied. Indeed, if $g: \bar{B} \rightarrow B$ is the factoring homomorphism and $\bar{A}$ is a $\beta$-partition of $\bar{B}$, set $A=g(\bar{A})$. Then $A$ is a disjointed subset of $B$ with cardinality $\leqq \beta$ such that $p(A)=h(\bar{A})$ is a cover of $B / I$, that is, the hypotheses of 
4.2 are satisfied. Applying this remark together with 3.6 and 4.3 gives

Corollary 4.4. A necessary and sufficient condition that a Boolean algebra $B$ satisfy $D_{\alpha \beta}$ is that there exist a complete field $\mathfrak{B}$, an $\alpha$-ideal $\Im$ of $\mathfrak{B}$, and a factorable, weak $\beta$-isomorphism of $B$ into $\mathfrak{B} / \Im$.

Another case in which $L_{\beta}$ is satisfied is where $I$ is an $\alpha \bigvee \beta$-ideal. For then every $\beta$-partition of $B / I$ is the image of a $\beta$-partition of $B$. (See the proof of 4.10 below.)

Corollary 4.5. Suppose $B$ is an $\alpha \bigvee \beta$-complete $D_{\alpha \beta}$-algebra and $I$ is an $\alpha \bigvee \beta$-ideal of $B$. If $\bar{B}$ is an algebra such that there is a weak $\beta$ isomorphism $h$ of $\bar{B}$ into $B / I$, then $\bar{B}$ is a $D_{\alpha \beta^{-}}$-algebra.

In particular, 4.5 applies if $I$ is the zero ideal. (Actually, a careful examination of the proof of 4.3 in this special case shows the completeness assumption on $B$ to be unnecessary and also that $\bar{B}$ is a $C_{\alpha \beta}$-algebra when $B$ satisfies $C_{\alpha \beta}$ and $h$ is a $\beta$-isomorphism.)

Corollary 4.6. Let $h: \bar{B} \rightarrow B$ be a weak $\beta$-isomorphism and suppose $B$ is an $\alpha \bigvee \beta$-complete $D_{\alpha \beta}$-algebra. Then $\bar{B}$ is a $D_{\alpha \beta}$-algebra.

Combining this corollary with 2.3 and 3.8 gives an interesting conclusion (which, in slightly different terms, is well known).

Corollary 4.7. A necessary and sufficient condition for a B.A. to be isomorphic to an $\alpha$-field of sets is that it be $\alpha$-complete and a $C_{\infty \alpha^{-}}$ algebra.

The result of Scott and Tarski [4] and Chang [2] now follows from $4.5,4.7$ and 3.10 :

Corollary 4.8. A necessary and sufficient condition that a Boolean algebra be $\alpha$-representable is that it be $\alpha$-complete and a $C_{\alpha \alpha}$-algebra.

This corollary, together with 2.3 and 4.6 gives the converse of 3.7 if $\beta \leqq \alpha$.

Corollary 4.9. Let $\beta \leqq \alpha$. A necessary and sufficient condition for a Boolean algebra $B$ to be a $\beta$-subalgebra of an $\alpha$-representable $B$. $A$. is that $B$ be $\beta$-complete and satisfy $C_{\alpha \beta}$.

As a particular case of 4.5 , we see that if $B$ is an $\alpha$-complete, $C_{\alpha \beta}$ algebra, where $\beta \leqq \alpha$, and if $I$ is an $\alpha$-ideal of $B$, then $B / I$ is a $C_{\alpha \beta}$ algebra. A slightly more refined result can be proved.

Corollary 4.10. If $\bar{B}$ is an $\alpha$-homomorph of an $\alpha+$-complete, $C_{\alpha \alpha+}$-algebra $B$, then $\bar{B}$ is a $C_{\alpha \alpha_{+}-\text {algebra. }}$. 
Proof. By 2.3 , it is enough to show that $\bar{B}$ is a $D_{\alpha \alpha+}$-algebra, and this conclusion will follow from 4.3 provided we can show that $L_{\alpha+}$ is satisfied.

Let $p$ be the given $\alpha$-homomorphism of $B$ onto $\bar{B}$. Suppose $\bar{A}$ is an $\alpha+$-partition of $\bar{B}$, say $\bar{A}=\left\{\bar{a}_{\xi}|| \xi \mid<\alpha+\right\}$, where $\xi$ runs over all ordinals of cardinality $<\alpha+$. For each $\xi$, choose $b_{\xi} \in B$ with $p\left(b_{\xi}\right)=\bar{a}_{\xi}$. Define $a_{\xi} \in B$ by $a_{\xi}=b_{\xi} \wedge\left(\bigvee_{\eta<\xi} b_{\eta}\right)^{\prime}$. Since $p$ is an $\alpha$-homomorphism and each $|\xi|$ is $\leqq \alpha, A=\left\{a_{\xi}|| \xi \mid<\alpha+\right\}$ is a disjointed subset of $B$ satisfying $p(A)=\bar{A}$. By $4.2, L_{\alpha+}$ is satisfied.

In some ways, Theorem 4.3 is unsatisfactory. To be sure, in many cases it is possible to verify the required condition $L_{\beta}$, as our various corollaries show. But there remain simple questions which are not covered by the theorem. For example, it is reasonable to hope that every $\alpha$-homomorph of a complete field is a $D_{\alpha \infty}$-algebra. The author has been unable to prove (or disprove) this conjecture. We do not even know of an example of an $\alpha$-representable algebra which does not satisfy $C_{\alpha \infty}$.

\section{REFERENCES}

1. G. Birkhoff, Lattice theory, Amer. Math. Soc. Colloquium Publications, rev. ed., vol. $25,1948$.

2. C. C. Chang, On the representation of $\alpha$-complete Boolean algebras, Trans. Amer. Math. Soc. vol. 85 (1957) pp. 208-218.

3. L. Loomis, On the representation of $\sigma$-complete Boolean algebras, Bull. Amer. Math. Soc. vol. 53 (1947) pp. 757-760.

4. D. Scott and A. Tarski, Metamathematical proofs of some Boolean representation theorems, (to appear).

5. R. Sikorski, $A$ theorem on the structure of homomorphisms, Fund. Math. vol. 36 (1949) pp. 245-247.

6. E. C. Smith, Jr., A distributivity condition for Boolean algebras, Ann. of Math. vol. 64 (1956) pp. 551-561.

7. E. C. Smith, Jr. and A. Tarski, Higher degrees of distributivity and completeness in Boolean algebras, Trans. Amer. Math. Soc. vol. 84 (1957) pp. 230-257.

8. M. H. Stone, Applications of the theory of Boolean rings to general topology, Trans. Amer. Math. Soc. vol. 41 (1937) pp. 375-481.

UNIVERSITY OF WASHINGTON 